\title{
3 Erster Anschluss: Metapher als Puzzle-Rätsel (Forceville)
}

Das Theoriemodell der Konzeptuellen Metapherntheorie ist erstmalig bei Charles J. Forceville als dezidierte Hintergrundfolie für Ausführungen zur Metapher im Kontext audiovisueller Bilder markiert. ${ }^{1}$ Beschäftigt sich Forceville mit der Metapher zunächst mit Blick auf das Format der Werbeanzeige im Printbereich, ${ }^{2}$ wendet er sich in der Folge zuvorderst dem Werbespot, ${ }^{3}$ später dann auch dem Animations ${ }^{4}$ und Spielfilm ${ }^{5} \mathrm{zu}$. Insbesondere die These der KMT, dass die Metapher ihrem Wesen nach kein genuin sprachliches, sondern ein kognitives Phänomen sei, ist leitend für die Untersuchung von Medien, die nicht ausschließlich oder nicht primär mit Worten, sondern maßgeblich mit Bildern arbeiten.

Anders als dies beim Folgekapitel (4 Metapher als Produkt und Automatismus) der Fall sein wird, begründet sich für die vorliegende Arbeit die Relevanz einer Erläuterung der Forceville'schen Metaphernauffassung jedoch nicht damit, dass eine Auseinandersetzung mit der zuvor skizzierten Verkörperungsthese der

1 Bereits bei Whittock wird die KMT kurz gestreift, sie ist jedoch nicht maßgebliche Fluchtlinie. Vgl. Whittock: Metaphor and Film, S. 113-118.

2 Charles J. Forceville: Pictorial Metaphor in Advertising. London/NewYork: Routledge 1996.

3 Charles J. Forceville: Bildliche und multimodale Metaphern in Werbespots. In: Zeitschrift für Semiotik 25.1-2 (2003), S. 39-60; Charles J. Forceville: Lecture 4. Pictorial and Multimodal Metaphors in Commercials. In: Online Cybercourse on Pictorial and Multimodal Metaphor 2006 (http:// semioticon.com/sio/files/forceville-metaphor/cforceville4.pdf, zuletzt eingesehen: 22.04.2017); Charles J. Forceville: Multimodal Metaphor in Ten Dutch TV Commercials. In: Public Journal of Semiotics 1.1 (2007), S. 15-34; Charles J. Forceville: Pictorial and Multimodal Metaphor in Commercials. In: Go Figure! New Directions in Advertising Rhetoric. Hrsg. v. E.F. McQuarrie und B.J. Phillips. Armonk: M. E. Sharpe 2008, S. 178-204.

4 Charles J. Forceville/Marloes Jeulink: The Flesh and Blood of Embodied Understanding. The Source-Path-Goal Schema in Animation Film. In: Pragmatics \& Cognition 19.1 (2011), S. 37-60, doi: 10.1075/pc.19.1.02for; Charles J. Forceville: Metaphor and Symbol. SEARCHING FOR ONE'S IDENTITY IS LOOKING FOR A HOME in Animation Film. In: Review of Cognitive Linguistics 11.2 (2013), S. 250-268.

5 Gunnar Theodór Eggertsson/Charles J. Forceville: Multimodal Expressions of the HUMAN VICTIM IS ANIMAL Metaphor in Horror Films. In: Multimodal Metaphor. Hrsg. v. Charles J. Forceville und Eduardo Urios-Aparisi. Berlin: Mouton de Gruyter 2009, S. 429-450; Thijs Renckens/Charles J. Forceville: The GOOD IS LIGHT and BAD IS DARKNESS Metaphors in Feature Films. In: Metaphor and the Social World 3.2 (2013), S. 160-179; Julius Koetsier/Charles J. Forceville: Embodied Identity in Werewolf Films of the 1980s. In: Image [\&] Narrative 15.1 (2014), S. 44-55; Charles J. Forceville: Visual and Multimodal Metaphor in Film. Charting the Field. In: Embodied Metaphors in Film, Television, and Video Games. Cognitive Approaches. Hrsg. v. Kathrin Fahlenbrach. New York/London: Routledge 2016, S. 17-32.

Ә Open Access. () 2020 Christina Schmitt, publiziert von De Gruyter. (G) BY-NC-ND Dieses Werk ist lizenziert unter der Creative Commons Attribution-NonCommercial-NoDerivatives 4.0 Lizenz. https://doi.org/10.1515/9783110614619-003 
KMT stattfinden würde. Denn in der Tat wird diese These in den im Folgenden zu betrachtenden Texten Forcevilles auch von diesem selbst aufgrund einer Fokussierung auf kreative Metaphern außen vor gelassen. (Es wird weiter unten noch darauf zurückzukommen sein, wie hier ,kreative' und ,konzeptuelle', mit Verkörperungsprozessen verbundene Metaphern als Gegenpole aufgemacht werden). ${ }^{6}$ Sich mit der Forceville'schen Position zur Metapher als kognitivem Phänomen im Kontext audiovisueller Bilder auseinanderzusetzen, ist vielmehr deshalb angezeigt, da in seinen Texten zur kreativen Metapher im Werbefilm ,Metapher im Zusammenhang mit einer Zuschaueraktivität gesehen wird. Im Kontext der Gesamtargumentation der vorliegenden Arbeit bildet Forcevilles Aktivitäts-Auffassung eine Abgrenzungsfolie: Vor ihrem Hintergrund wird sich im weiteren Verlauf das Verständnis der Aktivität des Metaphorisierens, wie ich es theoretisch und analytisch hier entfalten werde, klarer konturieren.

Es sei im Folgenden daher dargelegt, inwiefern bei Forcevilles theoretischem Modell die Metapher zwar als eine kognitive Zuschaueraktivität konzipiert ist (wie dies auch die vorliegende Arbeit tut), zugleich aber eine Auseinandersetzung mit der Körperlichkeit des Denkens nicht stattfindet. Dies rührt nicht nur daher, dass Image-Schemata und Primärmetaphern keine Rolle spielen: Vor allem ist das Metaphernverständnis von einem ,kalten“ Kognitionsbegriff durchdrungen, bei dem eine Erfahrungsdimension der Metapher unberücksichtigt bleibt. Denn die Metapher im Kontext des audiovisuellen Bildes ist bei Forceville letztlich als ein Puzzle-Rätsel konzipiert, oder, mit Blick auf die Aktivität des Zuschauers formuliert: Der Zuschauer löst ein Puzzle-Rätsel, indem er Metaphern identifiziert, konstruiert und interpretiert. Und letztlich erscheint die Metapher, aller Zuschaueraktivität zum Trotz, dadurch auch als etwas Statisches, bei dem Verkörperung keine Rolle spielt, und Dynamik höchstens im Sinne von Informationsverteilung relevant ist. Anders gesagt: ,Wahrnehmen' und ,Verstehen' werden im Sinne von Informationsaufnahme und -verarbeitung adressiert, und ,Fühlen` ist nicht von Belang.

Bereits der Medienbegriff, auf den sich Forceville stützt, deutet eine klassisch kognitivistische Ausrichtung an: ,Medium“ wird bestimmt als Träger und Transporteur von Informationen. Bezugnehmend auf einen solchen Medienbegriff, ${ }^{7}$

6 Vgl. zur Gegenüberstellung von kreativer und konzeptueller Metapher Renckens/Forceville: The GOOD IS LIGHT and BAD IS DARKNESS Metaphors in Feature Films.

7 Ohne dass eine Referenz für jenen Medienbegriff genannt wäre (und auch, wenn an anderer Stelle auf McLuhans „the medium is the message“ rekurriert wird), klingt darin das zwischenzeitlich vielfach kritisierte und dennoch prägend gewordene nachrichtentechnische Kommunikationsmodell von Shannon und Weaver an, das auf einem Sender, einem Empfänger und einem dazwischengeschalteten Medium mit der zu übertragenden Botschaft beruht (Claude Elwood 
wird das audiovisuelle Bild als multimodales Medium charakterisiert, insofern dort dem Zuschauer über mehrere Kanäle bzw. Signalsysteme Informationen überbracht werden: Visuelles, geschriebene Sprache, gesprochene Sprache, nichtsprachliche Geräusche und Musik werden als die fünf „channels“ bzw. „signaling systems“ bestimmt, mittels derer Filme Informationen transportieren und übermitteln. ${ }^{8}$ Einer solchen, recht technischen Medien- und Modalitätsauffassung korrespondiert eine Zeichnung einer mit der Metapher zusammenhängenden Aktivität, die dem Rational-Logischen verhaftet ist: Verhandelt wird hier eine rein kognitive Zuschauertätigkeit, welche ein Identifizieren (Schritt 1) sowie ein dadurch motiviertes Konstruieren und Interpretieren (Schritt 2) von Metaphern umfasst.

Zu (1) Metaphernidentifikation. Metaphern werden darüber identifiziert, dass Zuschauer auf entsprechende Hinweise reagieren, die vom Produzenten in einem audiovisuellen Bild platziert worden sind: „There must be a cue for the recipient of a commercial to link one thing metaphorically with something else." 9 Solche Hinweise, denen der Zuschauer nachgehen muss, zeigen dem Zuschauer die Notwendigkeit einer metaphorischen Rezeptionsweise an: „[V] iewers [are] alerted that a metaphor must or may be construed [...] they know that one thing (the ,target $t^{\text {) }}$ is presented in terms of a thing from another category (the ,source').“10

Als alarmierende Ursache und Motivation, einen Zuschauer $\mathrm{zu}$ einer solchen Metaphernidentifikation zu veranlassen, also dazu, zwei unterschiedliche Entitäten als Quell- und Zielbereich zu erkennen (d. h. als zwei miteinander in metaphorischer Weise zusammenhängende Kategorien bzw. Konzepte), werden nun vor allem Ähnlichkeit und gemeinsame Anwesenheit ausgemacht:

Target and source can each be represented visually, sonically, musically, or verbally (in spoken or written form) - or in a combination of these modes. Their conjoining is triggered by salient similarity (in the case of pictorial or other monomodal metaphors) or by simultaneous occurrence (in the case of multimodal metaphors). ${ }^{11}$

Shannon/Warren Weaver: The Mathematical Theory of Communication. Urbana: University of Illinois Press 1949).

8 Vgl. Forceville: Pictorial and Multimodal Metaphors in Commercials; Charles J. Forceville: NonVerbal and Multimodal Metaphor in a Cognitivist Framework. Agendas for Research. In: Multimodal Metaphor. Hrsg. v. Charles J. Forceville und Eduardo Urios-Aparisi. Berlin [u. a.]: Mouton de Gruyter 2009, S. 19-42, hier S. 21. An anderer Stelle spricht Forceville diesbezüglich auch von semiotischen Modalitäten (Charles J. Forceville: Pictorial and Multimodal Metaphor. In: Handbuch Sprache im multimodalen Kontext. Hrsg. v. Nina-Maria Klug und Hartmut Stöckl. Berlin/ Boston: De Gruyter Mouton 2016, S. 241-260).

9 Forceville: Pictorial and Multimodal Metaphor in Commercials, S. 193.

10 Ebd., S. 199, Herv. i. O.

11 Ebd., Herv. i. O. 
So können im audiovisuellen Bild zwei eigentlich disparate Dinge beispielsweise aufgrund audiovisueller Gestaltungsweisen verkoppelt werden, indem der Eindruck von Ähnlichkeit zwischen zwei Dingen suggeriert wird: „the film camera [...] can suggest resemblance between two things by filming them with the same unusual camera or lens movement“. ${ }^{12}$ Oder aber die visuell oder akustisch $\mathrm{zu}$ erfassende Repräsentation eines Quell- und Zielbereichs sind beide anwesend:

Imagine a cinematic shot of a giraffe, saliently accompanied by the trumpeting sound of an elephant in such a way that the trumpeting appears to emanate from the giraffe. Let us assume that the viewer-cum-listener believes that the filmmaker did not erroneously or whimsically give this impression, and that there is no good non-causal relation between the giraffe and the trumpeting sound (as would be the case, for instance, when the viewer understands the sound as being produced by an off-screen elephant that happens to trumpet at the same moment the camera shows the giraffe, for instance in a zoo). In such a case she would probably interpret the co-occurrence as intentional [...]. ${ }^{13}$

Der Zuschauer geht also Hinweisen nach, die vom Filmemacher für ihn intentional ausgelegt worden sind, um dem Zuschauer eine Botschaft zu übermitteln. Als Reaktion auf die Hinweise kann es dann zur Bildung der Hypothese kommen, dass die Information, wie sie vom audiovisuellen Bild übertragen wird, nur dann sinnstiftend ausgewertet werden kann, wenn eine Metapher konstruiert wird - „it is necessary/possible/useful to construe something as a metaphor“. ${ }^{14}$

Ob eine solche Hypothese aufgestellt wird, d. h. ob eine Metaphernidentifizierung erfolgt, hängt dabei auch immer davon ab, wie deutlich die Hinweise sind und ob es keine alternative Erklärung für die Gegenüberstellung zweier unterschiedlicher Entitäten gibt:

[...] there is a continuum from cases where metaphorical construal of two phenomena is virtually imperative because, in the given context, nothing but a metaphorical interpretation is an acceptable strategy to account for the odd juxtaposition of two phenomena, to cases where no metaphorical construal is necessary to make sense of this juxtaposition. In the latter case, the hints for metaphorical construal are so subtle that it occurs largely at the message addressee's own responsibility [.....$^{15}$

Zu (2) Metaphernkonstruktion und -interpretation. Ist die Metapher als solche erst einmal identifiziert, setzt das Konstruieren und Interpretieren der Metapher ein. Mit Blick auf die Rezeption der soeben zitierten Schilderung einer Filmein-

12 Forceville: Pictorial and Multimodal Metaphors in Commercials.

13 Forceville: Multimodal Metaphor in Ten Dutch TV Commercials, S. 17.

14 Ebd., S. 28, Herv. i. O.

15 Ebd. 
stellung heißt das: Das Giraffenbild und der Elefantenklang werden vom Rezipienten als Instanziierungen der Konzepte GIRAFFE bzw. ELEFANT aufgefasst, sodass er dann die (multimodale) Metapher GIRAFFE IST ELEFANT konstruiert und nach einer relevanten Bedeutung dieser Metapher gesucht wird (,search for a pertinent meaning“). ${ }^{16}$

Metaphern im Kontext audiovisueller Bilder $\mathrm{zu}$ konstruieren und $\mathrm{zu}$ interpretieren, heißt dann, dass Rezipienten nach jenen Aspekten suchen müssen, die vom konzeptuellen Quell- zum konzeptuellen Zielbereich übertragbar sind („search for mappable features“); ${ }^{17}$ sie müssen auswählen und entscheiden (,decide which facts and connotations adhering to the source domain [...] can be mapped onto the target domain“ $)^{18}$ und möglicherweise noch Anpassungen hinsichtlich der von ihnen getroffenen Auswahl vornehmen (,make appropriate adjustments to optimize the match between target and source“). ${ }^{19}$

Signifikant ist, dass Rezipienten für den Mapping-Prozess maßgeblich auf Wissen Bezug nehmen, das über den Rezeptionskontext des jeweiligen Spots hinausweist. Genauer: Der Bezug auf eine überindividuelle, nicht an den Kontext gebundene, systemische Konzept-Ebene erscheint für die Metaphernkonstruktion und -interpretation maßgeblich. Dies unterstreicht die argumentative Einbeziehung der Aristotelischen endoxa in das Metaphernmodell: Von der Summe der Fakten und Konnotationen, die dem Quellbereich anhaften - und aus denen der Zuschauer für das Mapping auswählt -, wird als der endoxa, der ,anerkannten Meinung“ gesprochen. ${ }^{20}$ In der Konsequenz heißt das, dass es wesentlicher Bestandteil des Mappings ist, das durch den Zuschauer das (unabhängig vom und vorgängig zum audiovisuellen Bild existierende) Wissen darüber eingebracht wird, was die bestehende endoxa eines Konzepts ist - ja, dieses Wissen ist gewissermaßen unverzichtbare Basis für das Mapping. Eine normative und systemische Bestimmtheit der Metapherninterpretation zeigt sich zudem auch in der großen Bedeutung, die dem Genre zugesprochen wird: „The genre within which a text (in whatever medium) is presented, or the genre to which it is attributed, determines and constrains its possible interpretations to an extent that is difficult to over-

16 Ebd., S. 17.

17 Forceville: Pictorial and Multimodal Metaphor in Commercials, S. 182.

18 Ebd., S. 179.

19 Ebd.

20 Vgl. hierzu ebd. Forceville bezieht sich hier auf Max Blacks Interaktionstheorie der Metapher, die auch für die vorliegende Arbeit (vgl. Kapitel 2) ein wichtiger Bezugspunkt ist (vgl. zur Interaktionstheorie Max Black: Metaphor. In: Proceedings of the Aristotelian Society. New Series 55 (1955), S. 273-294; Max Black: Mehr über die Metapher [1977]. In: Theorie der Metapher. Hrsg. v. Anselm Haverkamp. Darmstadt: Wissenschaftliche Buchgesellschaft 1983, S. 379-413). 
estimate [...].“21 Mit Blick auf das Werbegenre heißt das: Es ist von vorneherein die Erwartungshaltung des Rezipienten da, dass das beworbene Produkt positiv evaluiert werden soll. Und dies beeinflusst auch die Auswahl der übertragbaren Eigenschaften.

Die Metapherninterpretation, d.h. die Festlegung der Charakteristika des Quellbereichs, die zum Zielbereich zu übertragen sind, ${ }^{22}$ ist der eigentliche Konstruktionsakt der Metapher, und es ist ein Akt, der maßgeblich systemisch bestimmt ist. Denn das Mapping ist nicht über eine Beschreibung des audiovisuellen Bildes zu rekonstruieren, ${ }^{23}$ sondern ist vielmehr essentieller Teil des Interpretationsakts, mit dem der Zuschauer auf den „Stimulus“ reagiert, den das audiovisuelle Bild darstellt. ${ }^{24}$ Mit Blick auf die Giraffen-Filmeinstellung hieße das: Durch entsprechende Hinweise $\mathrm{zu}$ einer Metaphernidentifikation motiviert, legt der Rezipient dieser Einstellung in seinem mentalen Prozess fest, welche Eigenschaften des allgemeingültigen, überindividuellen Konzepts ELEFANT (repräsentiert durch den Elefantenklang) auf das Konzept GIRAFFE (repräsentiert durch das Giraffenbild) zu transferieren sind.

Die im Zusammenhang mit der Metapher stehende Aktivität ist somit als eine Zuschaueraktivität konzipiert, die einerseits auf der Verarbeitung medial transportierter Informationen beruht und andererseits auf dem Anwenden von Schemawissen gründet, das es ermöglicht, im Zusammenspiel mit den Interpretationsanweisungen logische Schlüsse zu ziehen - d. h. hier: sinnstiftende Metaphern zu bilden. Die Aktivität, so lässt sich sagen, wird infolgedessen als ein mentaler Prozess im Sinne der cold cognition entworfen, der die Kognitionswissenschaft gerade in ihren Anfängen verpflichtet war. Ohne dezidiert theoretische Bezugsfolie zu sein, resonieren in einem solchen Konzept daher auch nicht von ungefähr Positionen, wie sie in den 1980er Jahren von David Bordwells Initialschriften der sogenannten kognitiven Film- und Medientheorie aufgemacht wurden. ${ }^{25}$ Mit Nar-

21 Forceville: Non-Verbal and Multimodal Metaphor in a Cognitivist Framework, S. 32f.

22 Vgl. Forceville: Pictorial and Multimodal Metaphor in Commercials, S. 180.

23 Eben diese Auffassung - dass das audiovisuelle Bild selbst für die Mappingprozesse in den Blick zu nehmen ist - vertritt die vorliegende Arbeit. Vgl. Kapitel 9.

24 Vgl. Forceville: Pictorial and Multimodal Metaphor in Commercials, S. 181.

25 Vgl. David Bordwell: Narration in the Fiction Film. London: Methuen 1985; David Bordwell: Making Meaning. Inference and Rhetoric in the Interpretation of Cinema. Cambridge/London: Harvard University Press 1989. Alles in allem lässt sich bei den als ,Kognitive Film- und Medientheorie' gelabelten Forschungen, wie sie von Bordwell in den 1980er Jahren begründet wurden, eine Schwerpunktverlagerung beobachten, die auch für die Kognitionswissenschaft im allgemeineren Sinne Gültigkeit hat: „[...] cognitivist theorists in film and media studies have generally followed cognitive science's gradual move from a focus on ,cold' cognition (information-driven mental processes described in terms of inferential and computational models) to ,hot cognition“ 
ration in the Fiction Film und Making Meaning. Inference and Rhetoric in the Interpretation of Cinema hat Bordwell (insbesondere in Abgrenzung zur psychoanalytischen Filmtheorie $)^{26}$ die Idee eines aktiven Zuschauers prominent gemacht: Es ist der Zuschauer, der durch seine kognitiven Prozesse den filmischen Text erst hervorbringt. Das von Bordwell entworfene Modell von Zuschauerkognition, das auf eine Narration bezogen ist, beruht dabei wesentlich auf Informationsmanagement, Hypothesenbildung, logischen Schlussfolgerungen und inferenziellem Wissen: Zuschauer suchen nach Bedeutung (,scan the image in search of meaning، ${ }^{27}$ ) und bilden und überprüfen Hypothesen, was jeweils durch „cues unterschiedlichster Art und den verschiedensten Sorten von Schemata“ geleitet ist. ${ }^{28}$ In diesen Formulierungen Bordwells zeigen sich deutliche Parallelen zu denen Forcevilles. Und so erscheint auch Bordwells Auffassung, wie Zuschauer eines audiovisuellen Bildes durch Interpretation zu Bedeutung kommen, wesensverwandt mit derjenigen Forcevilles - nur dass jener von ,Metapher' redet, wo Bordwell allgemeiner von ,Bedeutung spricht: „in interpretation, meaning is arrived at through an interplay of conceptual schemes and perceived cues“. ${ }^{29}$

Zugespitzt formuliert heißt das: Nicht verkörpertes, sondern logisches Denken steht im Zentrum eines solchen Metaphernmodells. Infolgedessen zeigt

(affect-driven mental processes).“ (Ted Nannicelli/Paul Taberham: Introduction. Contemporary Cognitive Media Theory. In: Cognitive Media Theory. Hrsg. v. Ted Nannicelli und Paul Taberham. New York: Routledge 2014, S. 1-23, hier S. 5.) Firmieren primär Bordwells Monografien unter dem Label der cold cognition, so werden vor allem Publikationen von Smith, Tan und Grodal dem Label der hot cognition zugeordnet. (Murray Smith: Engaging Characters. Fiction, Emotion, and the Cinema. Oxford [u. a.]: Clarendon Press 1995; Ed S. Tan: Emotion and the Structure of Narrative Film. Film as an Emotion Machine. Mahwah: Erlbaum 1996; Torben K. Grodal: Moving Pictures. A New Theory of Film Genres, Feelings and Cognition. Oxford: Clarendon Press 1997.)

26 Vgl. für eine Rückschau auf das Verhältnis von kognitiver und psychoanalytischer Filmtheorie auch Vinzenz Hediger: Des einen Fetisch ist des anderen Cue. Psychoanalytische und kognitive Filmtheorie. Anmerkungen zu einem verpassten Rendez-vous. In: Film und Psychologie - nach der kognitiven Phase? Hrsg. v. Hans-Jürgen Wulff und Jan Sellmer. Marburg: Schüren 2002, S. 41-58.

27 Bordwell: Narration in the Fiction Film, S. 74; vgl. auch ebd., 103.

28 Vgl. David Bordwell: Kognition und Verstehen. Sehen und Vergessen in MILDRED PIERCE. In: montage AV 1.1 (1992), S. 5-24, hier S. 12, Herv. CS.

29 Bordwell: Making Meaning, S. 129. Nicht die Fragestellungen von Bordwell und Forceville seien hier einander gleichgesetzt. Denn obgleich beide an Kognition interessiert sind, differieren ihre Fragestellungen: Forceville beschäftigt sich mit der Frage, durch welche kognitive Aktivität Konsumenten, wenn sie Werbespots schauen, beworbene Produkte durch Metaphern wahrnehmen und sie deshalb positiv bewerten. Bordwell hingegen fragt danach, durch welche perzeptivkognitiven Aktivitäten der Zuschauer die Geschichte/Narration eines Films versteht bzw. konstruiert. Jenseits dieser Unterschiede zeigt allerdings die Art und Weise, wie über kognitive Prozesse nachgedacht wird, eine signifikante Ähnlichkeit auf. 
Forcevilles Ansatz auch Anschlussstellen zu aktuellen semiotisch-linguistischen Arbeiten auf, die das audiovisuelle Bild vor allem als logische Form adressieren $^{30}$ und den Textbegriff dafür stark machen. ${ }^{31}$ Bedeutung wird in solch Textzentrierten Arbeiten - und darin liegt ihre Verbindung $\mathrm{zu}$ Bordwell und Forceville - wesentlich auf eine textuell gelenkte Zuschaueraktivität zurückgeführt, bei der Rezipienten mit einem Text (mehr oder weniger) explizite „Interpretationsindizien“ zur Verfügung gestellt bekommen, aufgrund derer sie dann „gewisse sinnstiftende Anordnungen“ in einen Text hineinlesen können und sich ihnen ein Weg „zu einer vom ,Text“ präferierten Lesart“ erschließt. ${ }^{32}$

Das Fazit, das letztlich aus der Rede von der ,Metapher‘ als einer kognitiven Operation des Identifizierens, Konstruierens und Interpretierens gezogen werden kann, ist, dass Metaphern im Kontext audiovisueller Bilder wie Puzzle-Rätsel aufgefasst werden, die der Zuschauer zu lösen hat. Eine Metapher ähnelt hier in mancherlei Hinsicht dem Erfassen jenes Bildes, das sich zeigt, wenn man erfolgreich die Einzelteile eines klassischen Puzzle-Spiels zusammenfügt.

Ein Puzzle (ob nun ein einfaches für Kinder mit wenigen Teilen, oder aber ein komplexes für Erwachsene mit mehreren hundert Einzelteilen) existiert produktionsseitig als ein vollständiges Bild, das für den Spielenden in mehrere Teile geteilt wird. Als Puzzelnder hat man es daher mit einem ungeordneten Haufen unzusammenhängender Einzelteile zu tun. Das Puzzle-Bild ist also solange nicht vollständig erkennbar, bis die Einzelteile passgenau zusammengefügt sind. Dennoch wohnt diesen Bild-Fragmenten, eben weil sie zu einem Puzzle-Spiel gehören, immer schon das Potential ihres in sich stimmigen Gesamtbildes inne. Und so findet sich auch bei jedem Puzzle-Spiel auf dessen Verpackung eben das Gesamtbild, wie es sich aus den einzelnen Puzzleteilen zusammenfügt, immer schon abgebildet. Dieses Bild auf der Verpackung ist das Potential und die

$30 \mathrm{Vgl}$. John A. Bateman/Karl-Heinrich Schmidt: Multimodal Film Analysis. How Films Mean. New York/London: Routledge 2012; Janina Wildfeuer: Film Discourse Interpretation. Towards a New Paradigm for Multimodal Film Analysis. New York: Routlegde 2014.

31 Vgl. Janina Wildfeuer: Der Film als Text? Ein Definitionsversuch aus linguistischer Sicht. In: Film, Text, Kultur. Beiträge zur Textualität des Films. Hrsg. v. John A. Bateman, Matthis Kepser und Markus Kuhn. Marburg: Schüren 2013, S. 32-57.

32 Vgl. John A. Bateman/Matthis Kepser/Markus Kuhn: Einleitung. In: Film, Text, Kultur. Beiträge zur Textualität des Films. Hrsg. v. John A. Bateman, Matthis Kepser und Markus Kuhn. Marburg: Schüren 2013, S. 7-26, hier S. 11f. Den zuvor prägenden semiotischen Textbegriff, wonach ,Text ${ }^{\star}$ als ein „Produkt“ gefasst wird, das durch ,aneinandergereihte Zeichen“ bestimmt ist, erklären die Autoren damit für obsolet. Text sei vielmehr „ein essentiell dynamisches Phänomen“, insofern „Bedeutungen, Interpretationen und Wirkungen [...] erst im Austausch zwischen Text und Rezipienten“, d. h. im Zusammenwirken der Interpretationsindizien des Textes mit dem Hintergrundwissen und der Erfahrungswelt des Rezipienten be- und entstehen (ebd., S. 10). 
Intention der Einzelteile: Es zeigt sich darin das Versprechen, dass das richtige Zusammenfügen der Teile Sinn stiftet, ein Bild ergibt - dass es sich also lohnt, die einzelnen Teile überhaupt zusammenfügen zu wollen.

In vergleichbarer Weise, wie den Teilen eines Puzzlespiels ihr sinnvoller Zusammenhang von vorneherein gegeben ist, ist auch in der Forceville'schen Perspektive der metaphorische Zusammenhang zweier Dinge, die ein Film als disparate Dinge zeigt, bereits eine Gegebenheit. Denn zum einen ist die Metapher, die der Rezipient konstruiert, in der Regel vom Autoren/Produzenten intendiert und kalkuliert:

Makers' intentions are an important factor in the discussion about metaphor. [...] the construal of metaphors typically presupposes some authorial intention: the author usually wants a certain combination of perceptual elements to be understood as a metaphor [.... ${ }^{33}$

Eben an einer solchen Intention richten sich dann auch die Hinweise aus, die für den Rezipienten ausgelegt werden, um ihn zur Identifikation und in der Folge dann zur Konstruktion und Interpretation der Metapher zu bewegen. Doch jene Metapher, mit der ein Zuschauer einen Werbespot versteht, erscheint nicht nur durch die Autor-Intention als etwas Prä-Existentes (als etwas, das verschlüsselt wird, um seitens des Rezipienten dann wieder entschlüsselt zu werden). Denn sowohl Autoren/Produzenten audiovisueller Bilder als auch die Rezipienten dieser Bilder greifen letztlich auf die gleichen prä-existenten, sich auf einer überindividuellen, konzeptuellen Ebene befindlichen Schemata zurück.

Dass die Körperlichkeit des Denkens als metapherntheoretisch relevante Thematik in den hier vorgestellten Ausführungen Forcevilles ausgeblendet ist und die Metapher als ein kognitiv-rationaler Prozess des Puzzle-Lösens erscheint, mag auch dem Metaphern-Kontinuum geschuldet sein, das dem Forceville'schen Nachdenken über Metaphern zugrunde liegt. ${ }^{34}$ Denn konstatiert wird ein Kontinuum, an dessen einem Ende sich kreative, einmalige Metaphern befinden hier fungiert die Interaktionstheorie Max Blacks ${ }^{35}$ als metapherntheoretische Referenz. Am anderen Ende des Kontinuums wiederum befinden sich die tief in der konzeptuellen Struktur verankerten Metaphern - und nur hier fungiert die KMT als zentrale metapherntheoretische Referenz, und mit ihr also auch die These, dass Metaphern etwas mit Verkörperung zu tun haben. ${ }^{36}$

33 Forceville: Multimodal Metaphor in Ten Dutch TV Commercials, S. 16.

34 Vgl. Eggertsson/Forceville: Multimodal Expressions, S. 429.

35 Black: Metaphor; Black: Mehr über die Metapher.

36 Die KMT vertritt die Auffassung, dass auch kreative Metaphern letztlich auf das metaphorisch organisierte konzeptuelle System zurückzuführen sind: „[...] basic metaphors are part of those conceptual resources, part of the way members of our culture make sense of the world. Poets may 
Doch nicht nur, weil Image-Schemata und Primärmetaphern für Forceville aufgrund seines Fokus' auf kreative Metaphern keine Rolle spielen, ${ }^{37}$ ist das von ihm vorgebrachte Metaphernkonzept so statisch wie ein fertiges Puzzle-Bild. Zum einen findet das Mapping hier zwischen immer schon bestehenden Konzepten statt. Eine Verankerung in der Systemperspektive lässt sich zudem an dem konstatierten Metaphern-Kontinuum festmachen. Denn nur bezogen auf ein System lässt sich eine Aussage über die Qualität der Einmaligkeit bzw. des StrukturellVerankerten treffen; nur bezogen auf ein System ist zu beurteilen, ob man es mit einer kreativen Metapher oder aber mit einer konventionellen Metapher zu tun hat, geht es doch letztlich um die Frage, wie etabliert also die Metaphern hinsichtlich unseres konzeptuellen Systems sind. ${ }^{38}$ Eine Metaphernbetrachtung, die von einer Gebrauchsperspektive ihren Ausgang nimmt, ist hier nicht angelegt.

Und noch in einer anderen Hinsicht ist das Metaphernverständnis dem Statischen verhaftet. Denn die Zeitlichkeit audiovisueller Bilder findet nur sehr rudimentär Berücksichtigung. Eine Zeitlichkeit des Ausdrucks und des Wahrnehmens und Erlebens des audiovisuellen Bildes - seine Dauer, Expressivität und Entfaltung - gelangt nicht in den Horizont der Metaphernbetrachtung. Zeit spielt nur im Sinne von sukzessive aufeinanderfolgenden Zeitpunkten und dem damit einhergehenden Informationsmanagement eine Rolle. Anders als bei bewegungs-

compose or elaborate or express them in new ways, but they still use the same basic conceptual resources available to us all. If they did not, we would not understand them" (vgl. George Lakoff/ Mark Turner: More than Cool Reason. A Field Guide to Poetic Metaphors. Chicago: University of Chicago Press 1989, hier S. 25). Diese Überlegungen bleiben bei Forceville außen vor, wie auch dezidiert festgehalten wird (vgl. Forceville/Jeulink: The Flesh and Blood of Embodied Understanding, S. 38f.). Dennoch erscheint die KMT als zentrale Fluchtlinie für Forcevilles Metaphernauffassung und ist also auch für die kreativen Metaphern von Belang. Praktisch zeigt sich dies nicht nur daran, dass durchgängig KMT-Begrifflichkeiten wie Quell- und Zielbereich verwendet werden, sondern auch an der Typografie der Metaphernformulierungen: Gemäß KMT-Konvention wird zwischen KONZEPTUELLER EBENE (GIRAFFE IST ELEFANT) und Instanziierungsebene (Giraffe ist Elefant) unterschieden.

37 Zuletzt hat Forceville auch Metaphernanalysen in Image-Schemata-Perspektive vorgelegt. Siehe Charles J. Forceville: From Image Schema to Metaphor in Discourse. The FORCE Schemas in Animation Films. In: Metaphor. Embodied Cognition and Discourse. Hrsg. v. Beate Hampe. Cambridge: Cambridge University Press 2017, S. 239-256. Für eine Kritik und Diskussion der dortigen Analyse zu Ruth Lingfords kurzem Animationsfilm DEATH AND THE мотнER (GB 1997) vgl. Kappelhoff: Kognition und Reflexion, S. 112-117; Müller/Kappelhoff: Cinematic Metaphor, S. $22-29$.

38 Mit den Polen ,kreativ' versus, strukturell' wird damit eine Unterscheidung perpetuiert, wie sie schon die sprachwissenschaftlichen Metapherntheorien mit der Differenz ,poetisch`vs. ,konventionell` aufgemacht hatten. Mit ,kreativ` vs. ,strukturell` wird allerdings - ganz im Sinne der KMT - keine Aussage über die Lebendigkeit oder das Totsein von Metaphern angestrebt, beiden Metaphernarten wird vielmehr eine kognitive Realität zugesprochen. 
losen Bildern (wie z. B. bei einem Anzeigenplakat) können Quell- und Zielbereich einer konzeptuellen Metapher im Kontext des Bewegt-Bildes auch ungleichzeitig instanziiert werden: Die Bereiche müssen also nicht synchron, sondern können auch sukzessive, d. h. auch in verschiedenen Einstellungen gezeigt werden. ${ }^{39}$

Bevor jedoch im Rahmen der vorliegenden Untersuchung eine Gebrauchsperspektive entwickelt werden wird, die die Dynamik von Metaphern in mehrfacher Hinsicht berücksichtigt, sei die Aufmerksamkeit zunächst auf jene Arbeiten gerichtet, die dezidiert der Embodiment-These der KMT im Kontext audiovisueller Bilder nachgehen. Die Auffassung der KMT, dass ,Metapher‘ kein reines Sprach-, sondern im Grunde vielmehr ein kognitives Phänomen ist, ist auch dort wesentlich. Hinzu kommt nun, wo nicht mehr zuvorderst kreative, sondern tief verankerte Metaphern betrachtet werden, eine Beschäftigung mit dem Aufrufen körperlicher Erfahrung durch das Adressieren von Image-Schemata und Primärmetaphern. Die Dimensionen körperlicher Erfahrung, die der KMT zufolge Grundelemente komplexer, struktureller konzeptueller Metaphern sind, rücken somit ins Zentrum. Mit dieser Fokusverschiebung geht eine weitere signifikante Veränderung einher: Die Metapher wird nun nicht mehr mit Blick auf eine Zuschaueraktivität konzipiert, sondern in gewisser Weise sowohl als ein Produkt als auch als ein Automatismus verstanden.

39 Dies, so scheint es, ist dann auch der eigentlich signifikante Unterschied zwischen Metaphern im Werbespot und Metaphern in der Werbeanzeige. 